Cahiers de philosophie de l'université de

Levinas : au-delà du visible

\title{
Métaphore et hauteur
}

\section{Marc Faessler}

\section{OpenEdition}

Journals

Édition électronique

URL : https://journals.openedition.org/cpuc/827

DOI : $10.4000 /$ cpuc.827

ISSN : 2677-6529

\section{Éditeur}

Presses universitaires de Caen

\section{Édition imprimée}

Date de publication : 31 juillet 2012

Pagination : 143-160

ISBN : 978-2-84133-410-0

ISSN : 1282-6545

\section{Référence électronique}

Marc Faessler, « Métaphore et hauteur », Cahiers de philosophie de l'université de Caen [En ligne], 49 | 2012, mis en ligne le 07 juin 2018, consulté le 07 février 2023. URL : http://journals.openedition.org/ cpuc/827 ; DOI : https://doi.org/10.4000/cpuc.827

Creative Commons - Attribution - Pas d'Utilisation Commerciale 4.0 International - CC BY-NC 4.0 https://creativecommons.org/licenses/by-nc/4.0/ 


\section{Métaphore et hauteur}

Dans le propos sur l'au delà on soupçonne une métaphore emphatique de la distance intentionnelle. Même si dans ce soupçon on risque d'avoir oublié que le «mouvement» au delà, c'est la métaphore et l'emphase elles-mêmes, et que la métaphore, c'est le langage, et que l'expression d'une pensée dans un discours n'équivaut pas à un reflet dans le milieu indifférent d'un miroir, ni à une quelconque péripétie dédaigneusement appelée verbale et que le dire présuppose, dans le vécu de la signifiance, des relations autres que celles de l'intentionalité, lesquelles précisément, sur un mode non-récupérable, concernent l'altérité d'autrui: que l'élévation du sens par la métaphore dans le dit est redevable de sa hauteur à la transcendance du dire à autrui.

Levinas $^{1}$

ETTE CITATION RÉSUME EN PEU DE MOTS l'essence de l'éclairage
souverainement original apporté par Levinas à l'intrigue que nouent
entre eux - autour du mouvement pivot de la métaphore - langage, pensée de
la signification, hauteur et transcendance. Elle nous servira de repère dans le
décryptage des notes philosophiques et de la conférence inédite qui, dans les
deux premiers tomes des Euvres complètes, parlent de la métaphore et de ce
quil l'entoure ${ }^{2}$. Car "Herméneutique et au-delà»-postérieur d'une quinzaine

1. «Herméneutique et au-delà» [1977], in De Dieu qui vient à l'idée, $2^{\mathrm{e}}$ éd. revue et augmentée, Paris, Vrin, 1986, p. 166.

2. Sur la métaphore et ses entours, la liste des passages suivants peut être retenue dans la perspective de notre étude: a) Carnets de captivité, in E. Levinas, Carnets de captivité, suivi de Écrits sur la captivité et Notes philosophiques diverses (= CEuvres 1), R. Calin et C. Chalier (éd.), Paris, Grasset - IMEC, 2009, p. 131 sq. (le mot), p. 175 sq. (le symbole), p. 195 (transcendance de l'expression); b) Notes philosophiques diverses, in Euvres 1, Liasse A: $<1>$ p. 227-242 (métaphore), $<3>$ p. 248 et 250 (hauteur), $<4>$ p. 251-252 (responsabilité et idée de l'infini), $<8>$ p. 262-265 (sens et signification, hauteur), $<19>$ p. 267 (pouvoir métaphorique), $<30>$ p. 271 sq. (sens), $<32>$ p. 272-274 (Dieu), $<34>$ p. 276 sq. (Infini), $<95>$ p. 296 sq. (métaphore absolue), $<96>$ p. 297 (jeu de métaphores), $<97>$ p. 297 sq. (sens et langage), $<106>$ p. 301 (Dieu), $<111>$ et $<112>$ p. 302 sq. (misère, responsabilité), $<151>$ et $<153>$ p. 313 sq. (parole), $<157>$ p. 314 (Dieu), $<165>$ p. 317 (Infini), $<179>$ p. 322 (mots), $<190>$ p. 324 (langage, situation du nous), $<208>$ à $<210>$ p. 329-331 (métaphore); Liasse B: $:<4>$ p. 334 sq. (parole), $<16>$ p. 339 (langage diplomatique),$<48>$ p. $350-352$ 
d'années à la conférence sur la métaphore tenue au Collège philosophique en 1962 et paru trois ans après Autrement qu'être - atteste que la métaphore est restée dans la pensée d'Emmanuel Levinas un centre nodal de son approche du langage, même si le thème est en soi moins présent dans ses derniers écrits. Ainsi se laisse dessiner le plan qui peut guider notre lecture de ce que les Inédits contiennent concernant la métaphore: 1) repérer le mouvement au-delà de la métaphore en tant qu'essence du langage donnant sens à la «transcendance de jeu» de l'art littéraire; 2) élever le sens de la métaphore vers son transport éthique en repositionnant le langage face à la hauteur du visage de l'Autre; 3 ) entendre le mot Dieu comme métaphore des métaphores dans sa Transcendance d'Irrévélé. Ces trois angles d'approche permettant, en conclusion, d'évaluer l'impact de ces premières recherches sur la métaphore dans le développement d'ensemble de l'œuvre d'Emmanuel Levinas.

\section{Le mouvement de la métaphore}

La rhétorique classique du XVIII ${ }^{e}$ siècle aborde la métaphore comme la figure - le trope - d'un transfert de sens. «On transporte, pour ainsi dire, la signification propre d'un mot à une autre signification qui ne lui convient qu'en vertu d'une comparaison qui est dans l'esprit » ${ }^{3}$. Quand on parle d'une «source de chagrin», l'esprit établit intuitivement une similitude entre le ruissellement d'un point d'eau et le ruissellement des larmes. Cet élément qui relie implicitement le semblable au semblable en le transportant d'un contexte à l'autre constitue la métaphore dans sa figure rhétorique propre ${ }^{4}$.

(métaphore), $<50>$ et $<51>$ p. $352-354$ (Dieu), $<52>$ p. 354 (parler), $<53>$ p. 354 (idée de l'infini), $<60>$ p. 357-360 (pensée inséparable de l'expression), $<64>$ à $<67>$ p. 367-372 (extériorité antécédente du visage), $<69>$ p. 373 (nom et verbe), $<72>$ p. 375-377 (parole chez Heidegger et Merleau-Ponty), $<73>$ à $<75>$ p. 377-383 (la signification); Liasse C: $<23>$ à $<25>$ p. 393 sq. (parole, relation au visage), $<28>$ à $<30>$ p. 395 sq. (visage source de toute signification), $<90>$ p. 414 (le mot, essentiellement métaphore), $<92>$ p. 415 (langage), $<169>$ p. 437 (visage), $<177>$ p. 439 (Dieu), $<187>$ p. 442 (pensée = langage) $<229>$ p. 456 sq. (métaphore et enseignement oral), <239> p. 460 (langage); c) «La Métaphore. Collège philosophique le 26-2-62», in E. Levinas, Parole et Silence et autres conférences inédites au Collège philosophique (= Euvres 2), R. Calin et C. Chalier (éd.), Paris, Grasset - IMEC, 2011, p. 323-347.

3. Du Marsais (César Chesneau sieur du Marsais, 1676-1756), Traité des Tropes ou des différents sens dont on peut prendre un même mot dans une même langue [1730], Paris, Le Nouveau Commerce, 1977, p. 112. Chez Aristote, la rhétorique était partie intégrante de la philosophie. Elle s'en est émancipée pour former une discipline en soi, dont le déclin au XIX ${ }^{\mathrm{e}}$ siècle appelle de nos jours une reprise philosophique des questions laissées en suspens (voir P. Ricœur, La métaphore vive, Paris, Seuil, 1975).

4. Il permet de distinguer la métaphore: - de la métonymie (partie prise pour le tout, ou contenant pris pour le contenu: «des voiles sur le lac», «boire un verre»); - de la synecdoque (matière prise pour l'objet, ou caractéristique prise pour l'ensemble: "croiser le fer», «les mortels »); - de l'hypallage (attribution aux mots d'une phrase, par interversion d'un sens 
Du moins est-ce ainsi que la rhétorique classique la définit et la repère parmi les diverses figures de style.

Or Levinas va se pencher sur la métaphore en philosophe pour y déceler un enjeu beaucoup plus essentiel. Il cherche, dans le transfert de sens, à ressaisir le mouvement de pensée qui anime la métaphore. Ce mouvement n'est pas seulement la mise en rapport de deux contextes de signification en vertu d'un analogon. Car l'appel du semblable au semblable compare, mais ne donne rien à penser - reste pur effet de style. Le réel mouvement de la métaphore - celui qui va permettre de pointer en elle l'essence du langage - est une amplification qui mène la pensée plus loin, qui lui fait entendre une nouveauté qu'elle n'appréhendait pas - bref qui l'amène audelà. Cette amplification de la pensée - cette «emphase» comme aime à dire Levinas ${ }^{5}$ - se loge dans le «trans- » de la transfiguration du contenu initial d'un mot ou d'un verbe, prolongé en un sens figuré. «L'oiseau chante»! «La mort dans l'âme»! "Le ciel d'un regard»! Une élévation sémantique se produit, qui confère au vocable un miraculeux surplus de signifiance, un débordement du sens premier, un pouvoir de suggestion qui donne à penser. La métaphore se glisse "dans les "guillemets" qu'elle permet d'ajouter, dans le fait de se hausser sur la pointe des pieds, dans une espèce de lévitation - dans l'affirmation et un sens "autre" " ${ }^{6}$. Tout se passe comme si cette distance entre le sens propre d'un mot et son sens figuré rendait la métaphore nécessaire à la signification même qu'elle produit de par son dépassement du sens propre, lequel, à sa façon, y participe en écho. Il y a au creuset de la métaphore une sorte de bifurcation dont on peut présumer qu'elle révèle un caractère essentiel du langage. Levinas note:

Les diverses significations s'affirment dans une parenté mais quir n'est pas où une signification surgit dans l'autre par une véritable participation, comme si chaque signification pelait en quelque façon en laissant se détacher d'elle d'autres significations \{semblables à elle\} qưi $\{$ Elles $\}$ ne la reproduisent $<$ pas $>$ pas comme des enfants et comme si $\{\mathrm{Et}\}$ ces nouvelles pelures, toutes fines qu'elles étaient, pèlent encore, laissant tomber de nouvelles significations et que ces feuilles mortes de la signification recouvraient toutes les avenues du monde. \{Biffures de Michel Leiris\}?.

qui convient à d'autres mots de la même phrase: «rendre quelqu'un à la vie / rendre la vie à quelqu'un»); - toutes figures qui enjolivent le style, mais ne comportent pas le mouvement de transfert propre à la métaphore.

5. "La Métaphore», in Euvres 2, < f. 2 > p. 325.

6. Notes philosophiques diverses, liasse $\mathrm{B},<48>$ p. 350 .

7. «La Métaphore», <f. 16 > p. 335. Voir aussi: «Le pouvoir métaphorique du mot est dans le rapprochement de la signification et d'une autre signification dont elle est comme une pelure» (Notes philosophiques diverses, liasse $\mathrm{A},<1><\mathrm{f} .2$ verso $>$ p. 230). Et: «Le phénomène de 
La mention de Michel Leiris est très intéressante. Car dans un article paru dans Les Temps Modernes en juin 1949 sur la transcendance des mots, Levinas avait d'emblée souligné l'intérêt du procédé de Leiris qui, de bifurs (bifurcations) en biffures (corrections), invite la pensée à se séparer de la direction qu'elle semblait prendre et à cheminer vers l'inattendu, ce qui revient à «saisir la pensée au moment privilégié où elle vire en autre chose qu'elle-même ${ }^{8}$. Or les Notes philosophiques diverses de la liasse A s'ouvrent précisément en 1949 sur d'abondantes réflexions à propos de la métaphore. Il est raisonnable de conjecturer que l'originalité littéraire de Leiris a dû suggérer à Levinas une étude philosophique tout aussi originale sur la métaphore. Mais une telle étude - si le problème philosophique de la métaphore revient à la possibilité qu'aurait le langage d'entendre au-delà de ce que mesure la pensée - engage nécessairement un point de vue neuf sur l'essence du mot et du langage.

C'est ici que les Inédits sont révélateurs. Ils attestent de l'audace du geste philosophique de Levinas. Car - dépassant la tradition platonicienne $^{9}$, se dégageant implicitement de Husserl ${ }^{10}$, s'inspirant de Heideg-

la métaphore n'est pas dans la clarté qu'un terme reçoit d'un autre. Car pourquoi l'autre terme serait-il plus éclairant que le premier? C'est l'objet qui pèle. C'est ce miroitement, ce pellement $<$ sic $>-$ qu'est la signification (?)» (ibid., $<1><$ f. 4 recto $>$ p. 231).

8. "La transcendance des mots. À propos des "Biffures" de Michel Leiris», republié sous un titre abrégé in Hors Sujet, Montpellier, Fata Morgana, 1987, p. 216 (Poche, p. 198). Le texte de Biffures est aujourd'hui publié in M. Leiris, La règle du jeu, Paris, Gallimard (Pléiade), 2003, p. 1-285. Voir aussi l'article de P. Brunel, «Levinas: à propos de "Biffure(s)" ", in Le souci de l'art chez Emmanuel Levinas, D. Cohen-Levinas (dir.), Houilles, Manucius, 2010, p. 105-121.

9. Même si dans la tradition platonicienne la pensée est pure intellection ou contemplation et le langage son tombeau, «la célèbre définition de la pensée par Platon comme dialogue silencieux de l'âme avec elle-même - lie le langage à la pensée, lui enlève son caractère secondaire de simple expression d'une pensée pré-existante" (Notes philosophiques diverses, liasse A, $<28>$ p. 270). Dans le Cratyle: «le langage n'est pas simple ustensile, résultat d'une convention, il a un rapport avec la réalité qu'il imite» (ibid., $<97>$ p. 298 ; cf. $<1>$ $<$ f. 1 recto $>$ p. 227); et dans la $7^{e}$ Lettre: «la pensée exprimée est au-delà de la réflexion. Elle retrouve la métaphore» (liasse B, $<48>$ p. 351).

10. Dans «La signification et le sens» [1964] - texte qui reprend, parfois littéralement, de nombreuses notes sur la métaphore contenues dans les Inédits - Levinas se démarque de l'intellectualisme de Husserl. Ce dernier « rend compte des significations par un retour au donné. L'intuition catégoriale - notion par laquelle il rompt avec l'empirisme sensualiste prolonge, en réalité, l'intuitivisme de la signification. Les relations et les essences sont, à leur tour, données. L'intuition demeure la source de toute intelligibilité. Le sens est donné dans la droiture même qui caractérise la relation entre la noèse et le noème. La philosophie transcendantale de Husserl, n'est-elle pas une espèce de positivisme remontant, pour toute signification, à son inventaire transcendantal ? Les données hylétiques et les "prêts de sens" y sont minutieusement inventoriés, comme s'il s'agissait d'un portefeuille de valeurs. Même ce qui reste irréalisé, est donné, en quelque manière, en creux, dans une intention "signitive" ouverte, et s'atteste comme "effets impayés" dans le noème de la noèse. Toute absence a pour terminus a quo et pour terminus ad quem le donné. L'expression des significations ne 
ger $^{11}$ et de Merleau-Ponty ${ }^{12}$, mais suivant finalement son propre chemin phénoménologique - Levinas va défendre que le mot ne devient pas métaphorique, mais qu'il l'est d'emblée et qu'il dévoile ainsi la métaphore comme essence du langage.

Le mot ou le discours consiste non pas à évoquer un objet \{par un signe\}, mais à l'évoquer d'emblée en tant que ceci ou cela, c'est-à-dire en tant que significations - te transfert Ceci en tant que cela ou la métaphore n'est pas la modification apportée à the si une signification qui soi-disant existerait en dehors de tout langage; la métaphore qui aborde ceci en tant que cela est \{serait\} le phénomène premier de la signification ${ }^{13}$.

Le mot est d'emblée métaphore parce que toute expression se détache sur les horizons vécus d'un «monde» culturel, d'un contexte, d'une situation,

sert qu'à fixer ou à communiquer les significations justifiées dans l'intuition. L'expression ne joue aucun rôle ni dans la constitution, ni dans la compréhension de ces significations " (Humanisme de l'autre homme, Montpellier, Fata Morgana, 1972, p. 20-21 [Poche, p. 19]). Ce que confirme un texte ultérieur : "Chez Husserl - ou du moins chez le premier Husserl - la subreption et le glissement de sens (Sinnverschiebung) se produisent dans le langage, mais la pensée allant à l'être révélé "en chair et en os", a d'emblée une structure logique. Elle se reconnaîtra dans le langage» («Langage quotidien et rhétorique sans éloquence» [1981], in Hors Sujet, p. 210 [Poche, p. 191-192]).

11. Une longue note sur «le discours d'après Heidegger » souligne chez ce dernier une contemporanéité du parler silencieux avec le se-trouver-affecté (Befindlichkeit) et le comprendre (Verstehen). La fonction propre du discours «est originellement l'articulation même de la compréhension. Aux significations articulées s'ajoutent des mots - forme mondaine d'être, ayant le caractère de Zuhandenheit. L'articulation du monde - voilà le point qui pour Heidegger détermine tout le reste" (Notes philosophiques diverses, liasse $\mathrm{B},<72>$ p. 376 ; rappelons que Zuhandenheit renvoie à la signification «à-portée-de-main »). Et la conférence sur «La Métaphore» contient cette remarque: «Đe La priorité du sens figuré par rapport au sens dit simple - tel est le grand motif \{et la grande force\} des étymologies heideggeriennes. Il s'agit de remonter du sens concret et plat du terme, désignant souvent son essence technique, vers une signification procédant de toute une situation et qui ramasse en elle les possibilités d'objets nombreux qu'elle laisse se manifester» («La Métaphore», $<$ f. $16>$ p. 336). Mais pour Levinas le mot - certes lié à la sensibilité et aux horizons de la compréhension - renvoie moins à un «être-à-portée-de-main» qu’à un «être-à-portéede-métaphore» et à une "adresse-en-invocation-à-autrui».

12. Dans la phénoménologie de la perception de Merleau-Ponty, tout donné se place dans un horizon éclairé, y compris le mot qui reçoit le don d'être entendu à partir d'un contexte auquel il se réfère. La signification illumine toujours un horizon en accueillant - dans un geste créateur de la subjectivité - le déjà-fait historique du langage d'une culture, dès lors librement habitée. Le mot est un geste verbal: «[...] chez Merleau-Ponty le langage comme condition de la pensée - joue le rôle attribué au corps propre dans la perception. Ce qui est simplement Zuhandenheit chez Heidegger - devient corps chez Merleau-Ponty - pas instrument, mais corps, incarnation de la pensée. Toutefois aucun soupçon de la relation avec autrui. Autrui - c'est le monde dans lequel je suis plongé, une société où je suis engagé, comme je suis engagé dans mon corps. Je suis déjà engagé dans les autres pour parler avec les autres» (Notes philosophiques diverses, liasse B, $<72>$ p. 376 ).

13. «La Métaphore», <f. $14>$ p. 334 . 
que l'acte de signifier met en lumière sans épuiser la réserve de sens de l'horizon donné. Le mot signifie à partir de la position de celui qui regarde et qui, dans la prétention kérygmatique du langage, "évoque » ceci en tant que cela, c'est-à-dire à titre de signification. Le «ceci-en-tant-que-cela» est au fondement du mot la raison séminale d'où germera la métaphore - qui devient ainsi paradigme de toute métonymie ou synecdoque. Parler, s'exprimer par des mots, c'est toujours amener à la lumière un angle de vue, donc donner aux mots un sens d'emblée figuré au cœur de la signification évoquée, sens figuré gardant encore la capacité de désigner au-delà de ce qui est désigné - de transmuter le sens en d'autres sens. La signification que le mot porte produit sa propre capacité métaphorique. Le mot «bureau», par exemple, consacre l'être-à-portée-de-main de forme rectangulaire et de couleur marron sur lequel j'écris; mais déjà la spatialité solide à laquelle il renvoie lui donne la capacité de figurer - au-delà - l'horizon de la chambre où je travaille, et - au-delà encore - de devenir métaphore du «bureau de tabac» ou de l'expression «jouer à bureaux fermés » ${ }^{14}$. Aucun mot n'a de sens originairement littéral. Tous les mots de la langue s'ouvrent sur des possibles métaphoriques. Le langage quotidien en témoigne ( «élever les enfants», «se porter bien») et la poésie y puise son inspiration («les sanglots longs des violons de l'automne») ${ }^{15}$.

Ainsi donc, loin d'apparaître comme un phénomène exceptionnel du langage, comme une figure de style entre autres - la métaphore - déplacement de tout sens vers un autre, coïncide avec le phénomène même du langage et de la signification. [...]. Le langage est un système nouveau par rapport à celui qu'on aurait voulu voir dans la structure intentionnelle de la pensée - où chaque pensée est pensée de quelque chose. Le langage, c'est le fait que ce qui est pensé, ce noyau visé signifie, c'est-à-dire est déjà dépassé dans sa fixité, est en tant que quelque chose d'autre - est par conséquent métaphore ${ }^{16}$.

Levinas prend ici ses distances avec Husserl. Cela veut dire qu'à ses yeux la signification tient désormais à la métaphore, en tant que la métaphore est la participation même de l'objet à autre chose que lui - qui, par le mot,

14. Levinas donne l'exemple du mot «table»: meuble sur lequel on mange, bureau où l'on écrit, et, métaphoriquement, «table exécrable» de $\mathrm{M}^{\mathrm{me}} \mathrm{X}$ au sens des mauvais repas qu'on y sert («La Métaphore», <f. $12>$ p. 332).

15. Notes philosophiques diverses, liasse $\mathrm{A},<1><\mathrm{f}$. 9 recto $>$ p. 236. On peut souligner aussi le goût de Levinas pour les titres à ambiguïté métaphorique: Noms propres (noms de personnes/noms d'amis qui n'ont pas les mains sales); Hors Sujet (en marge de ce dont on parle/en extériorité du cogito); Entre nous («de vous à moi!»/en réciprocité sociale les uns avec les autres).

16. «La Métaphore», <f. $18>$ p. 337. 
creuse dans l'objet un sillon de sens. Ce qui lui permet de noter: « Le mot est essentiellement métaphore - il porte l'être au-delà de lui-même» et «être mot, c'est être plus qu'être, c'est être plus haut que l'être - c'est être métaphore ${ }^{17}$.

Cette dernière notation exprime toutefois tout le paradoxe du mouvement de la métaphore vers au-delà - adverbe lui-même métaphorique. Car si la métaphore est bien l'essence du langage amenant à l'expression l'ostension même de l'être dans un mouvement qui élève à la signification et donne sens en ouvrant un champ métaphorique à la parole, l' «au-delà » qui s'annonce en elle - cet élan de l'esprit vers le haut - ne représente pas pour le langage un passage vers l'altérité d'une Transcendance Tout Autre. Il dessine seulement la passe ontologique d'une «transcendance de jeu» qui ne laisse éclore et ne réhabilite que l'art. «La transcendance qu'ouvre l'art et d'une façon générale la culture - demeure métaphorique au sens où l'on dit "ce n'est qu'une métaphore" ${ }^{18}$. Car - contrairement à ce que l'on reproche parfois à la pensée de Levinas - l'art et la culture ne se résument pas pour lui à une sorte d'égarement dans lequel l'humain produirait de la beauté gratuite pour enrober la cruauté de son histoire ${ }^{19}$. Ils font originellement et en tant que tels partie de l'ordre ontologique. Ils en relèvent pleinement, rendant possible - par la littérature, la musique, la peinture ou l'architecture - une compréhension de l'être dans les divers profils de son multiple dévoilement. Ils sont bien de quelque façon «bergers de l'être»! Mais leur hébergement - par l'expression artistique assemblant l'être en significations - n'accueille, immanente, qu'une transcendance de pur jeu.

L'au-delà - ressaisi dans l'essence du langage au pli de ce «mouvement vers " propre à la métaphore - n'oriente-t-il en direction d'aucune autre hauteur, d'aucune autre et réelle Transcendance? N'est-il susceptible d'aucun retournement de la courbure spatiale des relations à autrui dans l'adresse de la parole? La métaphore de la hauteur va nous conduire sur un chemin inattendu.

17. Notes philosophiques diverses, liasse $\mathrm{C},<90>$ p. 414 et liasse $\mathrm{A},<1><$ f. 8 recto $>$ p. 235 .

18. "La Métaphore», <f. $19>$ p. 337-338.

19. En témoigne cette notation: «Il est facile de rappeler qu'en parlant nous dépassons constamment notre pensée, car nous utilisons un outil chargé d'une puissance d'évocation déposée en lui par l'histoire et par la société et que des significations harmoniques résonnent dès que le mot est proféré [...]. Car voici la parole devenue artiste: dans le système des signes verbaux $<$ f. $2>$ dont elle dispose, elle perçoit un clavier et le thème simple qu'elle avait à traduire tout d'abord s'enrichit de symphonies possibles" (Notes philosophiques diverses, liasse A, $<179><$ f. 1 - f. $2>$ p. 322 ; voir aussi $<8>$ p. 263). 


\section{Métaphore et hauteur}

Il est émouvant de découvrir au fil de ces notes philosophiques - qui pour la plupart ébauchent le futur contenu génial de Totalité et Infini - les hésitations, les scrupules, les objections que se fait à lui-même Levinas. On sait l'importance qu'a prise dans Autrement qu'être la distinction du Dire et du Dit et le fait que l'apophansis du Dire - ce «faire signe» pré-originel qui d'emblée répond d'autrui dans ce que met au jour le langage de son Dit signifie en tant que modalité de l'approche de l'autre et de la signifiance éthique du visage ${ }^{20}$. Mais il a fallu, pour le philosophe revenu de captivité, gagner pied à pied l'élaboration de cette pensée. Et c'est autour du motif de la «hauteur» - puisque la métaphore est apparue comme un mouvement irréductible menant l'esprit "au-delà», vers ce qu'il faut provisoirement nommer, dans son surplus de sens, le «haut ${ }^{21}$ - qu'un renversement imprévu s'est opéré. Mais ce ne fut pas sans quelques hésitations. Citons, en inversant l'ordre des pensées, les notes très denses d'une fiche datant de 1949-1950:

Je ne suis pas sûr que la métaphore - et le mouvement dans la signification vienne du fait que l'événement essentiel du langage est en-face-de-l'Autre. Comment concilier ma thèse: la parole dépossède celui qui parle et la thèse la métaphore est le dépassement de la signification? Comment montrer que le pouvoir du dépassement verbal se place dans la relation avec l'Autre ${ }^{22}$ ?

L'être et l'étant sont dans le langage en tant que déjà dit. [...] Par contre le langage qui se parle en face de l'Autre, toujours reprenant ce qui a été dit - est un mouvement infini, sans retour. Il ne se joue pas entre être et étant, mais en face de l'autre. Et c'est d'Autrui qu'il tient son pouvoir métaphorique [ce qui n'est pas sûr] ${ }^{23}$.

L'universalité du langage, c'est l'universalité de l'être et de l'étant. Parler, c'est dire l'être, dire ce qu'il en est de l'étant - Mais parler c'est aussi exercer un pouvoir métaphorique, transporter au-delà de l'être et de l'étant. De sorte que dans le langage il y a ce mouvement vers l'infini et il n'existe pas de langage sans ce mouvement. Et ce mouvement vient de l'autre, en tant que le langage est réponse à un autre et dépassement de ce qui est dit. [Cette dernière chose n'est pas sûre. Le dépassement de la métaphore ne vient-il pas de la trace ?] ${ }^{24}$

20. Autrement qu'être ou au-delà de l'essence, La Haye, Martinus Nijhoff, 1974, p. 6-9 et 29-76 (Poche, p. 16-20 et 43-99).

21. Voir Notes philosophiques diverses, liasse A, $<1><$ f. 5 recto $>$ p. 232.

22. Ibid.,$<1><$ f. 12 verso $>$ p. $241-242$.

23. Ibid.,$<1><$ f. 12 recto $-\mathrm{f}$. 12 verso $>$ p. 241 . Nous soulignons en gras.

24. Ibid., $<1><$ f. 12 recto $>$ p. 241 . Nous soulignons en gras. 
La difficulté consiste à orienter le «haut» par lequel la métaphore déborde tout sens, vers la «hauteur» d'un autre ordre de signifiance révélant - par-delà l'être et l'étant - ce qui justifie et donc rend possible la métaphore elle-même. Or, malgré ses hésitations et peut-être à cause d'elles, Levinas souligne dans le langage de la parole adressée à autrui un mouvement infini du dit et un mouvement vers l'infini du dire métaphorique. Comme si le mouvement vers le «haut», propre à la métaphore, avait à découvrir dans l'asymétrie où des interlocuteurs se parlent, le lieu abstrait par le truchement duquel le langage est placé dans la trace d'un Infini qui le justifie. Ce lieu, cette abstraction, c'est le visage. Il faut que l'infini, mouvementvers-le-«haut» propre à l'essence métaphorique du langage, rencontre la hauteur du visage pour que se noue l'intrigue éthique et transcendante qui rattache la vocation du langage à sa relation à l'Autre. "Hauteur distincte du vis-à-vis par contemplation, mais visage ${ }^{25}$.

Par le fait qu'en définitive toute parole est adressée à autrui, se produit une rencontre avec un interlocuteur - celui ou celle à qui l'expression exprime et qui, visage, n'est pas inclus dans l'ordre des significations se traduisant en mots. Dans cette rencontre survient donc un autre ordre de signifiance - hauteur asymétrique, non réciproque. Cet autre ordre de signifiance «- capable de la métaphore absolue dépassant la capacité du penseur est l'ordre qui rend seulement le langage des mots possible - qui est celui où luit le visage même auquel se tient le langage ", conclut la conférence sur la métaphore donnée en février $1962^{26}$.

Or en janvier de la même année, à la Société française de philosophie, Levinas présentait, devant un aéropage choisi et quelque peu médusé, les idées de Totalité et Infini. En voici un bref extrait:

L'épiphanie de l'Absolument Autre est visage où l'Autre m'interpelle et ordonne de par sa nudité, de par son dénuement. Il m'interpelle de son humilité et de sa hauteur. Il voit, mais reste invisible et, par là même, s'absout de la relation où il entre, reste absolu. L'absolument Autre, c'est Autrui. Et la mise en question du Même par l'Autre est une sommation de répondre. Le Moi ne prend pas seulement conscience de cette nécessité de répondre, comme s'il s'agissait d'une obligation ou d'un devoir dont il peut décider; il est, dans sa position même, de part en part responsabilité. Et la structure de cette responsabilité montrera en quoi Autrui, dans le visage, interpelle de très bas et de très haut - en ouvrant la dimension même de l'élévation ${ }^{27}$.

25. Ibid., $<4>$ p. 252.

26. "La Métaphore», <f. 26 > p. 342. Nous n’avons pas retenu la virgule proposée après "absolue».

27. "Transcendance et Hauteur", in Liberté et commandement, Montpellier, Fata Morgana, 1994, p. 66 (Poche, p. 79-80). Nous soulignons en gras (l'italique est de Levinas). 
Autrui appartient à la visibilité des phénomènes. Mais en tant que visage - on l'a souvent répété - il ne nous vient pas seulement dans les horizons d'un contexte, mais, sans cette médiation, signifie à partir de lui-même (kath'auto ${ }^{28}$. L'extériorité absolue est la condition de sa signification ${ }^{29}$. En ce sens, le visage n'apparaît pas. Il se dévêt de la phénoménalité où pourtant il signifie. Il s'absout du paraître dans lequel il est cependant vivante visitation. Le surplus d'où nous vient cette absolution, parle. Cette «parole» ne peut être entendue qu'au pli de métaphores dédisant la lumière du sensible tout en en montrant le sens. Nudité sans dénudement. Dénuement sans défense. Misère $^{30}$. Exposition à être. Humilité d'une hauteur. Extériorité d'une résistance éthique au meurtre ${ }^{31}$. Droiture qui commande: «ne m'anéantis pas» et «ne te fais pas d'image». La parole sans Dit - le Dire du visage - se signifie dans la trace de l'absolument Autre. Elle désarçonne la conscience, la bonne conscience du langage, en assignant à l'hypostase de tout sujet son renversement en altérité éthique, responsable de ce qui fait signe pour tous dans la visitation du visage. L'irrécusable de cet autre ordre de signifiance est un changement de position auquel nul humain ne peut se soustraire, car cette «transposition» le révèle dans la trace d'un Infini qui origine son altérité et lui désigne l'orientation éthique à laquelle sont désormais voués tout acte et tout langage.

Or ce retournement dans la vie du sujet doit être compris tel une kénose. L'extrait de "Transcendance et Hauteur » cité plus haut lie dans l'interpellation du visage "humilité» et «hauteur», «très bas» et "très haut», pour ouvrir à «la dimension même de l'élévation». Ce langage est proche de celui dont use le Talmud pour parler de la kénose ${ }^{32}$. Levinas l'emploie philosophiquement ici pour signifier que le «haut» vers lequel oriente l'«au-delà» de la métaphore est redevable - parce qu'il y a visage et altérité

28. Notes philosophiques diverses, liasse C, <130 > p. 426 : «Toute ma philosophie consiste à substituer au néant, à la négativité, à la néantisation - l'apparition d'autrui ». Cela signifie qu'à la dialectique sartrienne qui considère autrui comme un «trou dans l'être», se substitue l'apparition d'autrui comme épiphanie du visage.

29. Ibid., $<29>$ p. 395 : «Tout le passage où je montre que le visage est source de toutes significations doit être écrit ainsi : c'est l'extériorité qui est condition de la signification; signifier = être extérieur, mais présent».

30. Ibid., liasse A, <111 > p. 302-303: "Comme je ne veux pas fonder la relation sur l'universalité du langage, mais sur sa relation - (car l'universalité démolit les termes et aboutit à l'unité) - il me faut qualifier cette relation : la misère».

31. Ibid., liasse $\mathrm{B},<64>$ p. 367 : «Le fait qu'autrui résiste à mon meurtre éthiquement, non pas comme une force, mais comme visage - accomplit mon rapport avec l'extérieur».

32. Voir «Judaïsme et kénose» [1985], in À l'heure des nations, Paris, Minuit, 1988, p. 133-151. Sur une possible interprétation d'Autrement qu'être comme expression du concept de kénose, voir M. Faessler, "Kénose», in Emmanuel Levinas et les théologies, D. Cohen-Levinas et S. Trigano (dir.), Paris, In Press (Pardès; 42), 2007, p. 143-153. 
de tous et pour tous - d'un évidemment du sujet qui n'a pas le temps de faire retour à soi. Il s'agit donc d'une véritable kénose du cogito, seule à même de réordonner - à partir de cette responsabilité d'une obligation infinie mais non encore accomplie - la parole et son langage ${ }^{33}$. La "transcendance de pur jeu» des métaphores se voit ainsi mise en question et réorientée. La prétention du discours qui s'exempte de toute expression nécessaire à la bonté envers les créatures est désormais abaissée. À l'inverse, le manque - orientant un dire en réponse au dénuement, à la privation, voire à la misère de l'homme sans Dieu - est souverainement élevé comme ce qui doit guider le langage dans ses diverses approches du réel. L'humilité de la parole et la hauteur de l'exigence morale se conjoignent pour regarder le monde d'où nous regarde le visage.

Le visage - le noumène, le kath'auto - se tourne vers moi comme nudité et misère et, par là, s'intègre dans le monde par rapport auquel Dieu est transcendant (?).

Mais son apparition $\{($ de l'Autre qui n'est pas Dieu $)\}$ n'est pas une apparition à moi - il n'est pas pour moi. L'expression comme bonté, est bonté pour tous. Dans le Toi qui pénètre dans le monde s'annonce lui et eux. Le visage nous regarde - il n'est pas comme l'objet vu par tout le monde - il regarde tout le monde. Je regarde celui qui regarde tout le monde. [...]

L'essentiel de ma conception. Le discours n'est pas impersonnel. Il repose sur la bonté. Je parle à quelqu'un ${ }^{34}$.

Désormais la métaphore peut être «en-visagée» comme un mouvement qui porte le discours au-delà de celui qui le tient et dont le sens figuré « est le sens qu'un terme prend dans un contexte humain: là où l'objet par le langage est offert à Autrui ${ }^{35}$. La hauteur qui donne sens à l'usage des métaphores et à l'être qui se révèle à travers elles n'est pas verticale mais humble - éthique à hauteur de visage, essentiellement bonté. Du coup elle rend indirectement à l'art ses lettres de noblesse, le renvoyant à ce vers quoi l'être est orienté: l'infini dans la trace duquel la relation à l'autre se tient dans l'en-face du visage. Orientation dont la littérature, par le jeu de ses métaphores, exprime entre les lignes - en creux ou en contestation - le mi-dire ${ }^{36}$.

33. Notes philosophiques diverses, liasse A, $<160>$ p. 315 : «L'Autre est la condition d'une liberté qui vide la Moi de sa pesanteur".

34. Ibid., liasse $\mathrm{C},<169>$ p. 437 .

35. Ibid., liasse $\mathrm{B},<48>$ p. 351 .

36. L'essence de l'art littéraire est de signifier entre les lignes. Il appelle l'exégèse. Comme si chaque œuvre n'était jamais que la fable d'un entre-dit ineffable. Dans son article «Exercices sur "La folie du jour" " [1975], Levinas - qui a certainement lu le texte de son ami Blanchot à sa parution en 1948 (cf. Carnets de captivité, < p. 53 (1948) > p. 188) - sait que la fiction du 


\section{Dieu - métaphore des métaphores}

Le transport propre à la métaphore peut être maintenant abordé sous l'angle de la nouveauté que représente, au cœur de la signification du sens, le mouvement de pensée épousant un sens qui excède ce qui est pensé. Levinas considère en effet les mots «Dieu» et «au-delà», ou l'expression "absolument Autre», comme des métaphores. Mais ce que désignent ces métaphores n'est pas un contenu. Ce qui est visé dans les intentions de pensée qui les portent, c'est certes le mouvement propre à la métaphore mais en tant que pensée-pensant-plus-qu'elle-ne-pense. Or ce débordement qui déloge le pensé de la pensée rejoint exactement la situation du renversement de position du sujet mis en question par le réquisit du visage, où il reçoit - d'une extériorité à laquelle il ne peut se dérober mais qu'il ne peut pas non plus amener à la mesure de sa pensée - l'unicité d'une élection de soi l'enjoignant à «répondre de... », c'est-à-dire à répondre... de ce surcroît inassimilable.

À cause de ce surcroît inassimilable, nous avons appelé la relation qui rattache à lui le Moi, idée de l'infini. L’idée de l'infini est la métaphore par excellence. L'idée de l'infini consiste précisément et paradoxalement à penser plus que ce qui est pensé $\{$,$\} enle \{$ łe conservant eependant dans la démesure par rapport à la \{de ce qui est $\}$ pensé. L'idée de l'infini consiste à saisir l'insaisissable en lui garantissant cependant son statut d'insaisissable. S'ily avait dans $\{$ Si $\}$ l'idée de l'infini, elle ne serait plus idée de l’infini $<$ sic $>$. Et cependant si le surplus ou le surcroît

narrateur «au pied du mur» où il doit être fusillé et qui ne l'est finalement pas, dissimule un vécu réel de l'auteur, résistant capturé par les nazis. Mais il veut interpréter le récit. Et il décrit en introduction comment il conçoit l'abord de la littérature: «Que l'expression ne vienne pas s'ajouter à la pensée; que - métaphore - elle porte cette pensée au-delà du thème pensé; que par-delà ce thème - encore transmissibles, comme un marteau ou comme un document -, les lettres - dans leur déploiement, dans leur littérature - gardent les raisons séminales du Dit et promettent à l'interprète, c'est-à-dire au lecteur, un sens plus lointain et plus ancien ou plus profond, un sens inspiré - c'est cela, sans doute, l'Intelligibilité ellemême. De soi - et non seulement pour un esprit fini - elle demande écrivains et lecteurs, de soi elle exige le Livre» (Sur Maurice Blanchot, Montpellier, Fata Morgana, 1975, p. 55). Levinas interprète ensuite La folie du jour comme la fable de la fermeture de l'être qui de soi étrangle l'humain dans une interminable agonie, autrui - seul point où pourrait s'ouvrir un dehors - n'ayant plus d'issue. Fable où se dénonce la «folie d'Auschwitz qui n'arrive pas à passer", "l'infernal. L'infernal qui se montre à Auschwitz mais qui se tapit dans la temporalité du temps et la maintient» (p. 6o). Dans les Notes philosophiques diverses, liasse C, $<92>$ p. 415 , cette notation: «Blanchot: Le langage se situe avant le rapport avec autrui - dans une étrangeté de soi à soi. Moi: Le langage - c'est l'Ansprächen $<$ sic $>-$ l'invocation. La reconnaissance d'autrui comme tel». Et $<70>$ p. 406-407: «Dans l'art de Blanchot la réalité devient vraiment fantomatique». Sur la relation et l'amitié entre Levinas et Blanchot, voir Emmanuel Levinas - Maurice Blanchot, penser la différence, É. Hoppenot et A. Milon (dir.), Nanterre, Presses universitaires de Paris 10, 2007. 
insaisissable ou impensable ne concernait en rien le pensée visant un thème $\left\{\right.$ Moi\}, il n'y aurait pas eu davantage d'idée de l'infini ${ }^{37}$.

Ce fragment raturé, dans lequel la pensée cherche sa meilleure expression, est essentiel. Le mot infini y est écrit avec une minuscule. Ce qui veut dire que dans la position désarçonnée du sujet - relation sans mesure face à l'extériorité absolue du visage - l'insaisissable vient à l'idée en tant qu' «idée de l'infini» s'inscrivant par sa démesure même dans le «mouvement audelà » pensant-plus-qu'il-ne-pense propre à la métaphore.

Levinas note encore: "La merveille des merveilles de la métaphore, c'est la possibilité de sortir de l'expérience, de penser plus loin que les données de notre monde. Qu'est-ce que sortir de l'expérience? Penser Dieu ${ }^{38}$. L'idée de l'infini qui nous vient de la relation à Autrui comme visage consiste donc à penser le surcroît irrévélé du mot «Dieu» dans la visée de signification d'une structure de pensée métaphorique, débordée par la Transcendance visée mais lui garantissant ainsi l'absolu de son Altérité. Le mot Infini peut alors s'écrire avec majuscule et la relation à autrui prendre le sens de « religion» - ce qui relie à l'Impensable.

Dans les Inédits et avant même qu'il affine son analyse de «l'infini en nous » cartésien, Levinas parvient par sa réflexion sur la métaphore à une approche de Dieu sans corrélat intentionnel - ce qui déplace le Transcendant vers le corrélatif de la justice et du juste langage que les humains ont à bâtir entre eux.

La métaphore absolue de la transcendance uniquement si le transport consiste en soumission éthique. [...] La soumission à l'infini qui est l'idée de l'infini - c'est-à-dire un sens par-delà la capacité et l'identification - ne peut être qu'un acte direct incapable de retour - ce en quoi réside sa force et non pas sa faiblesse $[\ldots]^{39}$

Les amarres sont donc rompues avec toute pensée de l'Être divin qui viserait à thématiser et englober son objet - meublant ainsi des arrière-mondes. Dieu - Transcendance du Transcendant - doit relever du dérangement de l'ordre, de l'extériorité irréversible, de l'irrectitude intentionnelle. On attendrait, pour le «dé-nommer», le mot d’Illéité. Il n’apparaît pas dans les Inédits ${ }^{40}$, alors que l'idée de trace fait déjà son chemin. En revanche émerge l'expression: "Dieu est l'Irrévélé " ${ }^{41}$. Cette formulation est très

37. "La Métaphore», <f. 28 > p. 344.

38. Notes philosophiques diverses, liasse $\mathrm{A},<1><\mathrm{f} .4$ recto $>$ p. 231.

39. Ibid., < $95>$ p. 296-297. Nous avons rétabli «uniquement» pour «unique».

40. Sauf peut-être dans cette notation sibylline: «Lui - quand il apparaît entre deux, dans leur intimité» (ibid., liasse C, $<204>$ p. 447).

41. Ibid., liasse $\mathrm{B},<51>$ p. 353 . 
intéressante car, dans sa note, Levinas la lie à l'orientation du désir sans défaut dont la gratuité d'action n'est pas assurée d'aboutir - ce qui est l'exact statut de la conscience dans l'usage de la métaphore et de la pensée vouée à penser-plus-qu'elle-ne-pense en embrassant l'idée de l'Infini. Que Dieu soit l'Irrévélé vient donc éclairer des formules telles que «la métaphore des métaphores - Dieu» ${ }^{42}$, ou "la métaphore par excellence est Dieu» ${ }^{43}$. Formulations étonnantes qui rappellent la conclusion d'une étude sur Roger Laporte datant de 1966 - «le langage, c'est le fait qu'un seul mot toujours se profère: Dieu ${ }^{44}$ - et qui annoncent déjà la dernière phrase d'Autrement $q u$ 'être ${ }^{45}$. On doit les comprendre comme une élévation hyperbolique de la métaphore vers ce qu' «irrévèle » à la pensée, sous la guise du commandement éthique, la hauteur du visage.

Sans métaphore on ne peut pas entendre la voix de Dieu. Sans métaphore toute pensée serait ce qu'elle est et signifierait en fin de compte ce qui correspond à la capacité de la pensée.

Mais il n'y a peut-être pas de voix de Dieu?

Il faut retourner la réflexion: sans Dieu il n'y aurait pas de métaphore. Dieu est la métaphore même du langage - le fait d'une pensée qui se hausse au-dessus d'elle-même (Ce qui ne veut pas dire que Dieu n'est qu'une métaphore. Car il n'y a pas d'autre métaphore que le mouvement qui porte vers Lui $<$ ) $>{ }^{46}$.

Si Dieu - l'Irrévélé - est la métaphore même du langage, si - par la séparation non pensable de sa Transcendance - Il est, sans être Lui-même métaphore, la voix de fin silence qui perce la visée métaphorique du langage en l'appelant de sa Hauteur, c'est que Dieu doit être entendu, au cœur de la parole, comme Créateur. Il est Transcendance créatrice qui se retire du don du langage où se produit la simultanéité du présent dans le dévoilement métaphorique de l'être, mais qui - par ce Retrait que le visage signifie en portant l'investiture du Créateur ${ }^{47}$ - dévoile la métaphore à elle-même dans l'ultime élévation qui la hausse à sa responsabilité éthique et la porte

42. Notes philosophiques diverses, liasse $\mathrm{A},<1><\mathrm{f} .12$ recto $>$ p. 240 .

43. Ibid., $<1><$ f. 8 verso $>$ p. 236.

44. « Roger Laporte et la voix de fin silence» [1966], in Noms propres, Montpellier, Fata Morgana, 1976, p. 137.

45. " [...] la destitution et la dé-situation du sujet ne restent pas sans signification: après la mort d'un certain dieu habitant les arrière-mondes, la substitution de l'otage découvre la trace - écriture imprononçable - de ce qui, toujours déjà passé - toujours “il” - n’entre dans aucun présent et à qui ne conviennent plus les noms désignant des êtres, ni les verbes où résonne leur essence - mais qui, Pro-nom, marque de son sceau tout ce qui peut porter un nom» (Autrement qu'être ou au-delà de l'essence, p. 233 [Poche, p. 284]).

46. Notes philosophiques diverses, liasse $\mathrm{A},<1><\mathrm{f} .6$ recto $>$ p. 233.

47. Ibid., liasse C, $<215>$ p. 451 : «Ce qui a une signification par soi-même c'est le visage. Il porte l'investiture du Créateur. Le visage est la créature par excellence». 
vers l'insaisissable de l'Infini. Dieu est métaphore des métaphores sans être simple métaphore, parce que sa Transcendance - par sa percée dans la relation au visage d'autrui - se produit comme désir dans le mouvement de la métaphore et de la pensée. Désir de tourner l'intension métaphorique du penser vers l'Altérité du Très-Haut qui, de par son dérobement même, la justifie - la retourne en injonction de justice. Et désir concomitant de ne pas aborder le prochain les mains vides, en orientant le langage - qui sans cesse offre le sens du «monde» à autrui - vers une bonté qu'inspire notre statut de créatures. Paradoxe de l'Irrévélé, dans un retournement de tout athéisme et un exhaussement de la métaphore «Dieu»: «L'homme est Dieu pour l'homme » ${ }^{48}$ ! Au cœur de la parole, Dieu, métaphore par excellence, ne comble pas le langage humain qui le vise, mais l'astreint à la générosité envers les autres. Le Créateur est l'optique éthique de la créature.

Les notes, fragments et esquisses des Inédits nous amènent là.

On peut se demander, en guise de conclusion, quels prolongements «inédits » ces recherches sur la métaphore ont produit dans l'œuvre ultérieure de Levinas. Sans être exhaustif, on peut signaler deux thèmes qui, en lien avec ce qui vient d'être pensé à propos de la métaphore, s'approfondissent: l'idée de l'Infini et l'emphase.

Durant les années où ont été rédigées les fiches des Inédits, Levinas a déjà publié sur l'idée de l'Infini et celle-ci jouera un rôle essentiel dans Totalité et Infini ${ }^{49}$. Mais il y est revenu en 1975 dans une contribution vraiment innovante intitulée «Dieu et la philosophie " ${ }^{\circ}$, fruit d'une suite de conférences données au moment où paraissait Autrement qu'être. Or cette nouvelle approche - qui en soi lie «idée de l'infini» et «signification d'une pensée vouée à penser-plus-qu'elle-ne-pense » ressaisie au creux de la métaphore - fait glisser cette dernière vers l'idée de passivité. La rupture du mouvement de la métaphore vers l'insaisissable - rupture reprise dans la démesure par laquelle l'idée de l'Infini-en-nous met en œuvre une cogitatio (une noèse) dont le cogitatum (le noème) échoue à accomplir la visée, et qui demeure débordée, en tant que cogitatio, par son objet - est maintenant décrite comme un traumatisme suggérant la passivité du créé. «La rupture de l'actualité de la pensée dans l' "idée de Dieu", est une passivité plus passive que toute passivité; comme la passivité d'un traumatisme sous

48. Ibid., $<110>$ p. 420

49. "La philosophie et l'idée de l'Infini » [1957], in En découvrant l'existence avec Husserl et Heidegger, Paris, Vrin, 1967, p. 165-178. Le texte fait partie d'une section intitulée «Raccourcis»!

50. «Dieu et la philosophie» [1975], in De Dieu qui vient à l'idée, Paris, Vrin, 1982, p. 93-127. 
lequel l'idée de Dieu aurait été mise en nous ${ }^{51}$. Ce qui, dans le mouvement au-delà de la métaphore, la haussait vers l'altérité, vers le Très Haut, vers l'inatteignable du Créateur, la replie désormais vers l'en deçà an-archique - sans fondement autosuffisant - d'un cogito non seulement désarçonné mais débouté de lui-même en sa passivité de créature. L'intrigue de sens qui se noue dans l'idée de l'Infini enrôle donc la métaphore dans l'évocation de cet en deçà indéclinable - éveil à un "plus » dans le moins ${ }^{52}$ en contrepoint $\mathrm{du}$ «surplus» vers le haut propre au mouvement métaphorique. Comme si le ne-pas-se-laisser-englober de «l'idée de Dieu en nous» était aussi une non-indifférence de la Transcendance pour la pensée, donc pour le langage de la métaphore. Le mouvement de la métaphore d'abord voué à la signification d'un penser-plus-qu'on-ne-pense se voit désormais affecté à un penser-autrement-qu'on-ne-pense. L'autrement qu'être est à portée de métaphore!

Si cette analyse est fondée, elle éclaire l'emphase hyperbolique dont use Autrement qu'être ou au-delà de l'essence au tournant de métaphores créant un style philosophique unique en son genre. Or, dans la même année 1975, Levinas fut invité par l'Université de Leyde à répondre aux questions d'un aréopage de professeurs sur l'ensemble de son œuvre ${ }^{53}$. Parmi les réponses qu'il improvise librement, l'une porte sur cette affirmation d'Autrement qu'être ou au-delà de l'essence: "La philosophie n'est peut-être que cette exaltation du langage où les mots - après coup - se trouvent une condition à laquelle les religions, les sciences et les techniques doivent leur équilibre de sens ${ }^{54}$ - allusion limpide, semble-t-il, à la signifiance empruntée au pouvoir métaphorique des mots. Dans sa réponse - qui contient plusieurs niveaux - Levinas va s'attacher à justifier une autre manière de passer d'une idée à l'autre qu'il définit ainsi: «passer d'une idée à son superlatif, jusqu'à son emphase. Voici qu'une idée nouvelle - nullement impliquée dans la première - découle ou émane de la surenchère " 55 . C'est l'application du mouvement de sens de la métaphore à la signification de l'idée! Le déplacement de sens s'y fait seulement par transmutation ou sublimation d'une

51. «Dieu et la philosophie», p. 106.

52. Ibid., p. 110-111, passage qui cite en note Michée, 1, 3-4 et s'inspire de ses métaphores: «Profondeur d'un subir que ne comprend aucune capacité, que ne soutient plus aucun fondement, où échoue tout processus d'investissement et où sautent les verrous qui ferment les arrières de l'intériorité. Mise sans recueillement, dévastant son lieu comme un feu dévorant, catastrophant le lieu, au sens étymologique du terme. Éblouissement où l'œil tient plus qu'il ne tient; ignition de la peau qui touche et ne touche pas ce qui, par-delà le saisissable, brûle. Passivité ou passion où se reconnaît le Désir ».

53. "Questions et réponses» [1977], in De Dieu qui vient à l'idée, p. 128-157.

54. Autrement qu'être ou au-delà de l'essence, p. 228 (Poche, p. 278).

55. "Questions et réponses», p. 141-142. 
idée à l'autre selon la modalité de l'exagération, c'est-à-dire du passage métaphorique à la limite. Exemple: «quand je dis: La passivité consiste à se livrer, à subir au-delà de toute passivité, d'une passivité qui ne s'assume pas, j'aboutis à la fission du soi ${ }^{56}$. L'enseignement de la métaphore est réassumé au service de la vérité du discours philosophique. Et Levinas défend avec hardiesse sa méthode:

Je traite, vous le voyez, de l'emphase comme d'un procédé. Je pense y retrouver la via eminentiae. C'est en tout cas la manière dont je passe de la responsabilité à la substitution. L'emphase, cela signifie à la fois une figure de rhétorique, un excès de l'expression, une manière de s'exagérer et une manière de se montrer. Le mot est très bon, comme le mot «hyperbole»: il y a des hyperboles où les notions se transmuent. Décrire cette mutation, c'est aussi faire de la phénoménologie. L'exaspération comme méthode de philosophie ${ }^{57}$ !

L'amplification de pensée de la métaphore était qualifiée d'emphase par Levinas dans sa conférence au Collège philosophique ${ }^{58}$. Ici l'emphase est rapprochée de la via eminentiae qui permettait à l'ontologie scolastique de passer - selon un chemin d'analogie - des êtres formels à l'Être divin suréminent. Mais Levinas en détourne le sens. L'analogie est remplacée par l'excès dans le langage, l'expression poussée à l'extrême, la métaphore hyperbolique, qui favorise la transmutation du sens des mots vers des signifiances d'autrement qu'être ${ }^{59}$. La voie d'éminence ouvre à la pensée l'au-delà de l'être.

L'«autrement», dégagé au cœur de l'usage métaphorique du mot, atteste, en définitive, que l'entier de l'œuvre lévinassienne se veut visage de la métaphore.

Marc FAessler

Genève

56. Ibid., p. 142.

57. Ibid.

58. «La Métaphore», <f. $2>$ p. 326 : «- la métaphore \{semble $\}$ indiquer une amplification de la pensée, \{une emphase\} haussant, en quelque façon, le ton, s'épurant et se sublimant, tout en demeurant dans le contenu même dont elle part et qu'elle transfigure».

59. Exemple à propos du soi-même qui ne peut se faire, car il est déjà fait de passivité absolue: «Les verbes, les adjectifs possessifs et les figures syntaxiques dont on voudrait user pour désarticuler la singulière torsion ou contraction du soi-même, réfugié ou exilé dans son propre plein jusqu'à l'éclatement ou la fission - en vue de sa reconstitution en guise d'identité identifiée dans le Dit - portent déjà la marque du soi-même, de cette torsion, de cette contraction, de cette fission" (Autrement qu'être ou au-delà de l'essence, p. 132 [Le livre de poche, p. 165]). La métaphore est ainsi comme innée à l'autrement qu'être de l'ipséité du sujet. 